\title{
Making Models of Measurement Tools: Examples from Think-Aloud Student Interviews
}

\author{
Benjamin M. Zwickl ${ }^{*}$, Dehui Hu* Noah Finkelstein ${ }^{\dagger}$ and H. J. Lewandowski ${ }^{\dagger, * *}$ \\ * School of Physics and Astronomy, Rochester Institute of Technology, Rochester, NY 14623 \\ ${ }^{\dagger}$ Department of Physics, University of Colorado Boulder, Boulder, CO 80309 \\ ${ }^{* *}$ JILA, University of Colorado Boulder, Boulder, CO 80309
}

\begin{abstract}
Constructing and using models are core scientific practices that have gained significant attention within K-12 and higher education. Although modeling is a broadly applicable process, within physics education, it has been preferentially applied to the iterative development of broadly-applicable principles (e.g., Newton's laws of motion in introductory mechanics). We show how similar modeling processes can be invoked as a means to understand the real-world complexities of experimental apparatus, including the measurement tools, in upper-division laboratory courses. In the context of a think-aloud experimental activity involving optics and electronics, we document examples where students apply all of the key facets of modeling to their apparatus and measurement tools: construction, prediction, interpretation of data, identification of model limitations, and revision. A modeling perspective reframes many of the seemingly arbitrary technical details of measurement tools and apparatus as an opportunity for authentic and engaging scientific sense-making.
\end{abstract}

Keywords: physics education research, laboratory, experimental physics, modeling, assessment PACS: $01.40 . F k, 01.40 . g b, 01.50 . P a, 01.50 . \mathrm{Qb}$

\section{INTRODUCTION}

Scientific practices, a set of habits of mind and skills for scientific inquiry and reasoning, are gaining increasing prominence in science education alongside the traditionally valued core disciplinary ideas [1]. In particular, models, abstract representations of real-world systems that describe, explain, or predict certain aspects or behaviors of the physical world, are becoming an explicit part of the curriculum. Modeling, by which we mean constructing models, using models to make predictions or interpret data, testing models against observations, and refining models, is highlighted in the Next Generation Science Standards [2] and in several existing curricula in active use in high schools [3] and colleges [4, 5, 6]. Most of these introductory physics courses employ the practice of modeling to develop students' ideas about fundamental principles of physics (e.g., Newton's laws of motion). As such, most explicit frameworks for modeling focus on the development of principles, but rarely address how models can be used to understand a complete experimental apparatus, inclusive of the measurement tools. We argue that in upper-level physics lab courses it is essential to take a broader view of modeling that includes the entire apparatus in the modeling process. Specifically, we show that any framework that is intended to describe upper-division students' model-based reasoning in a laboratory setting should include models of the measurement tools. We provide a simplified framework for modeling that has been employed elsewhere for laboratory curriculum design [7], and show examples of upper- division students employing various facets of modeling to their measurement tools as part of an upper-division lab activity.

Before going into the new framework and examples of upper-division student reasoning, it is worth contrasting introductory labs, where the goal is often making a quick connection to fundamental principles, with upperdivision labs, which are closer to authentic physics research in their complexity and instrumentation. Introductory labs commonly employ two techniques to quickly elucidate physical principles. First, many introductory lab experiments are engineered to make the behavior of the system agree with highly simplified models that are easily derived from principles. The experimental details are arranged so as not to distract students' attention from the principles. For instance, the canonical "block sliding down a plane" is commonly implemented using a cart with wheels. The knife edge wheels are designed to have low rolling resistance, are mounted to low friction axles, and their low mass and radius result in a small moment of inertia that ensures the rotational kinetic energy in the wheels is small compared to the translational kinetic energy of the cart. One manufacturer explicitly states how the carts are designed to ensure that "student data more closely matches theory." [8] The second technique employed in many introductory labs is the use of sensors and automated data acquisition [6,9]. The various sensors do an excellent job of quickly obtaining quantitative data and providing real-time graphic visualizations of the data. With such tools, students can quickly compare predictions to measurements, make refinements to their 
models, and run a test again. However, the principles of operation and performance limitations of the measurement tools are rarely discussed. The measurement tools are engineered so that any deviation from ideal measurement operation can be ignored when used for common introductory experiments. Further, the model of the measurement tool is often integrated into the apparatus and software, so every sensor directly outputs the quantity of interest. For example, force sensors output newtons while position sensors output meters even when those quantities are not directly measured. Students are encouraged to focus on the relationship between force and motion rather than on how these measurements work or on random uncertainty and systematic errors of the sensors.

While prioritizing basic physics principles is natural for an introductory course, upper-division physics majors need a more holistic view of the experimental process. This holistic view should include a deep understanding of the principles of physics underlying the entire apparatus, including the measurement tools. In a recent survey about laboratory learning goals, faculty frequently mentioned the importance of understanding the "black boxes" in the laboratory through an understanding of the principles of operation and limitations of operation [10]. Modeling the full apparatus affords an important professional development opportunity for students pursuing careers in physical science or engineering. Physicists, as a community, are frequently pushing the limits of commercially available measurement tools and are often on the leading edge of designing new measurement techniques (e.g., Magnetic Resonance Imaging), which is only possible through a deep intellectual engagement with the entire experiment. What is needed is a extension of existing modeling frameworks $[11,12]$ that is more applicable to the complex apparatus of upper-division labs.

\section{MODELING FRAMEWORK FOR LABS}

Frameworks for describing modeling at the introductorylevel emphasize the development of new principles [11], while the measurement process is largely taken for granted. In upper-division physics courses, it is much less common to inductively develop new fundamental principles (e.g., Maxwell's equations), but very common to apply known principles to explain observable phenomena. Although the principles may be provided, students' work is directed at constructing an abstract model, identifying key principles and parameters, making relevant assumptions, and making predictions that are testable. Because many upper-division labs involve advanced concepts and use equipment that is often unfamiliar, students must do significant intellectual work in order to understand the experiment.

The holistic framework for modeling in the laboratory in Fig. 1 gives equal attention to both the physical system

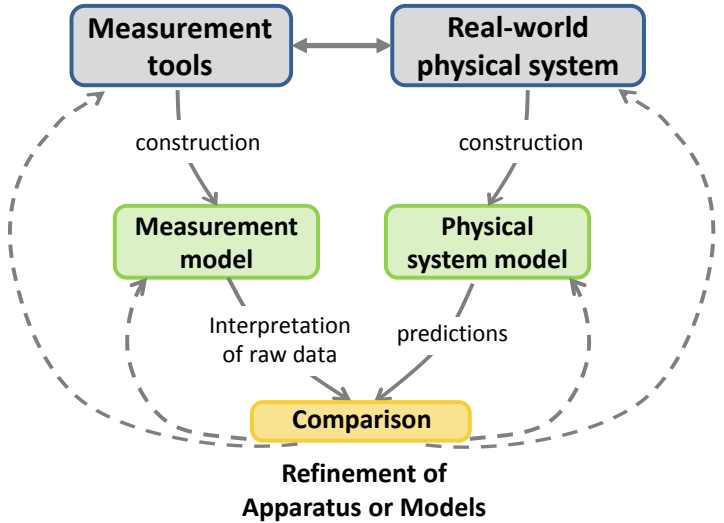

FIGURE 1. A framework for modeling that includes the physical system and measurement tools.

and the measurement tools. The full experimental apparatus is conceptually divided into a physical system (e.g., a block sliding down a ramp) and the measurement tools (e.g., a motion detector). The symmetry of the framework emphasizes that the measurement tools represent another system amenable to deep understanding, whose mechanistic operation is rooted in principles of physics that fully explain how the tools work. Like any model, a model of measurement tools represents some idealized behavior and articulates limits on the range of situations where the idealized behavior occurs. Further, the model of the measurement tools is essential for translating raw observations into usable data comparable with theory. Like all models, it is subject to revision, which is often referred to as a calibration. The process of constructing a model of a measurement tool requires identifying principles of operation, key device parameters, and limitations on ideal performance. While this model construction process is usually aided by documentation from manufacturers, it still bears all the features of model-based reasoning. It is also worth noting that the key step of comparing data and theory now takes place within a larger set of modeling activities. Also, any revision of the experiment could include a revision of a model or apparatus of either the measurement tools or the physical system.

\section{THINK-ALOUD LAB ACTIVITIES}

Based on extensive conversations with experimental physicists, we are confident that modeling measurement tools is an essential practice for researchers. However, we wanted to document examples of such reasoning in a fairly typical undergraduate upper-division laboratory activity. To that end, eight physics majors in their 3rd-5th years of study volunteered to participate in an individual think-aloud laboratory activity that typically took 30-45 minutes. All participants were enrolled in an upper-level optics and modern physics lab course that typically en- 


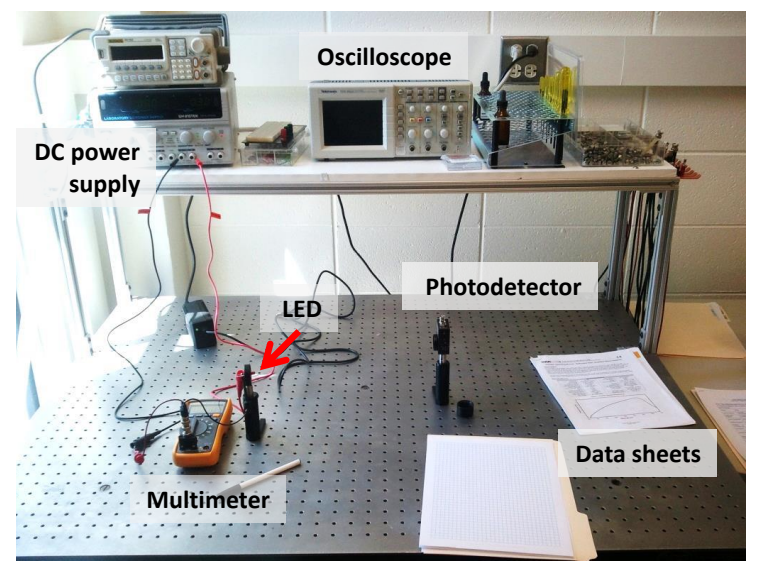

FIGURE 2. Laboratory bench used for the think-aloud experimental activity.

rolls about 25 students at a large state university. The activity was conducted in the lab space at the end of the semester and involved equipment (shown in Fig. 2) that was used by the students in several experiments throughout the semester. The lab activity was based on an activity that students had completed in a prerequisite lab course. The short prompt for the activity was:

"You have been hired by an LED manufacturer to run quality control tests of the optical power output of their new high efficiency red LEDs. Answer all the questions on the graph paper provided:

1. What is the predicted total power emitted by the LED based on the manufacturer's specification?

2. What is your measured total power emitted by the LED?

3. How do the measured and predicted powers compare?

4. What assumptions and approximations were included in your prediction or measurement?

5. What could you do to improve the comparison?"

The physical system consisted of a red LED connected to a DC power supply in series with a resistor to limit the maximum current through the LED. A multimeter was used to measure current through the LED. The measurement tools used to determine the optical power output were a Thorlabs PDA36A Adjustable Gain Photodetector and an oscilloscope. Students were also provided with data sheets for the LED, photodetector, multimeter, and oscilloscope along with assorted optical mounts. The experiment was designed to provide students with opportunities to construct and refine models of the LED and the photodetector using several approaches, levels of sophistication, and simplifications. Audio and video of the think-aloud interview were collected along with written observations and copies of students' work.

\section{RESULTS}

The interviews were transcribed and coded for instances of model-related activity (e.g., construction, interpretation of data, prediction, revision, etc.) and whether the student was attending to features of the physical system, the measurement tools, or both. The modeling codes were primarily drawn from a more detailed modeling framework designed for upper-division laboratory courses [7]. Two coders achieved agreement exceeding 95\% after a refinement of the coding criteria. The first result is that modeling and using the measurement tools occupied a significant amount of time in the activity. In the three interviews that were analyzed in full, students spent about 30-50\% more time modeling and using the measurement tools (photodetector and oscilloscope) than modeling and using the physical system (LED). In addition to demonstrating that measurement tools occupied a large quantity of students' time, the following excerpts give examples of the quality of students' modelbased reasoning. Examples are provided that span four different aspects of modeling in relation to the measurement tools: construction, identification of assumptions and limitations, interpretation of data, and revision. The main claim that follows from these examples is that any framework for model-based reasoning that ignores the use of measurement tools may be missing significant episodes of model-based reasoning, which are especially likely to occur in upper-division physics labs that involve sophisticated measurement equipment.

Model construction: Identifying principles of operation A student is trying to understand the physical mechanism by which the incident light on the detector is related to the output voltage $V$ being measured with an oscilloscope. The student realizes that the broadly dispersed light intensity must be integrated over an area to get a total incident power $P_{i n}$. The power from the light produces a current (according to a material-dependent proportionality constant $R$, known as the responsivity). The current is converted to a voltage using a circuit known as a "transimpedance" amplifier, which has gain $G$ specified in volts/amp. Mathematically, $V=G R P_{i n}$.

[Student A] "I've got to figure out how to turn voltage measurements into power. I guess I don't have any idea how to do that honestly. (Student is reading data sheet) Tell me power to voltage...voltage to power... spectral responsivity. I kind of remember this stuff. Something like when light [is] incident on the photo detector and that intensity is integrated into a power and that power causes a current and the current transimpedance gain... So I have voltage and I'm looking for a power. This is figuring out power from a measured voltage." The student goes on to successfully construct a quantitative model connecting a 
measured voltage to an incident power. However, by first identifying the principles of operation, the mathematical equation that is used is not just a computational tool, but has been clearly linked to the physical setup.

Identifying limitations of the measurement tools and appropriately designing the experiment [Student B] The student recognizes and addresses two limitations in the measurement tools and apparatus. First, the LED emits light over a range of angles, but the photodetector's small size may not be large enough to capture the entire cone of light. The effect of the limitation can be minimized by bringing the detector as close as possible to the LED. Second, the student observes that ambient light is also incident on the detector, the effect of this limitation can be reduced by blocking more ambient light.

[Student B] "I think I'm just gonna put the photodiode directly in front of the LED and hope that it's in between the 23 degrees of the viewing angle so I get approximately 100 percent of the relative intensity...I just put the photodiode close so, that makes sure that I get as much of the intensity as I can. I'm gonna put this [light blocking tube] on to eliminate a lot of the sunlight."

Using the measurement model to interpret data In this excerpt, a student constructs a model of the measurement tools, complete with actual device parameters for the peak wavelength of the LED, responsivity of the photodetector at the peak wavelength, and transimpedance gain. The desired measurement (optical power output of the LED) is only possible when this full mathematical model is properly constructed and used to interpret the raw voltage measurements.

[Student B] "I know the voltage is proportional to the intensity. I know the photodiode has got a small diode in there and it's only picking up a small area of intensity from the LED so, that's the area, if I knew that area and multiplied it by I, I'd get watts. Power equals I times A. I'm still struggling to figure out how I'll get voltage into intensity. Once I have intensity, I can get the power. I know volts are watts per amp...I know I need to use this responsivity which is an amp per watt in the peak wavelength for the LEDs is 635 [nm], so 635 on this gives me a responsivity of 0.4 amps per watts. So I need to get this into power. So, one over responsivity gives me watts per amp so, I need to calculate the current from the photodiode. I'm gonna use the gain which is at $0 \mathrm{~dB}$ setting so the gain is volts per amp which I believe is $1.51 \times 10^{3}$ volts per amp. That times the responsivity of 0.4 gives us volts per watt which is 604 volts per watt, so one over that gives me the wattages per amp er... per volt. ...So, now multiplying our $\Delta V$ which is $22.2 \mathrm{mV}$ times this watts per volt gives us the intensity as seen in the photodiode. I get a power of $3.84 \times 10^{-5} \mathrm{~W}$. That was the power, the measured power from the photodiode."

Revision of apparatus and model A student revises the apparatus to minimize ambient light and revises the model of the photodetector to include an offset in the photodetector output voltage that is present even when the LED is off (i.e., $V=G R P_{\text {in }}+V_{\text {off }}$ ). This kind of revision of a measurement model is often referred to as a calibration measurement.

[Student B] "It looks like the photodiode is definitely detecting [light]. Okay the photo diode is detecting a lot of sunlight. It's detecting the diode but the sunlight is definitely affecting the reading I'm just trying to think of the best way to get it. I think I'm just gonna put the diode right up against this thing to minimize all the stray light.It looks like with the LED off there's an offset voltage of just under $20 \mathrm{mV}$.... With the LED on, the voltage goes up to $39.2 \mathrm{mV}$....The difference is $23.2 \mathrm{mV}$ from the diode being on to off. Now, we're gonna turn the voltage output into total power emitted by the LED."

\section{CONCLUSIONS}

In support of the goal of integrating scientific practices, such as modeling, across the physics curriculum, we must highlight such practices whenever they are likely to occur. The examples from the think-aloud experimental activities demonstrate that measurement tools support rich opportunities for model-based reasoning, and that any framework that describes modeling in the laboratory must necessarily consider the full apparatus, inclusive of measurement tools, when trying to describe students' reasoning. We hope our framework and results encourage additional lab curricula to embrace modeling as a theme that naturally weaves conceptual and mathematical reasoning into upper-division lab courses.

The authors would like to thank the CU Physics Department for input on learning goals and pointing out the importance of modeling measurement tools. This work is supported by NSF TUES DUE-1043028 and NSF TUES DUE-1323101.

\section{REFERENCES}

1. National Research Council, A Framework for K-12 Science Education, Tech. rep. (2012).

2. Next Generation Science Standards (2013), URL http://www.nextgenscience.org.

3. M. Wells, et al., Am. J. Phys. 63, 606 (1995).

4. E. Etkina, et al., "Investigative Science Learning Environment," in Reviews in PER Vol. 1, AAPT, 2007.

5. E. Brewe, Am. J. Phys. 76, 1155 (2008).

6. D. R. Sokoloff, et al., Eur. J. Phys. 28, S83 (2007).

7. B. M. Zwickl, et al., Am. J. Phys. 82, 876-882 (2014).

8. PASCO, PasCars Dynamic Carts (2014), URL http://www.pasco.com/prodCatalog/ME/ME6950_pascar-set-of-2/\#featuresTab.

9. R. K. Thornton, et al., Am. J. Phys. 58, 858 (1990).

10. B. M. Zwickl, et al., Am. J. Phys. 81, 63 (2013).

11. D. Hestenes, Am. J. Phys. 60, 732 (1992).

12. C. V. Schwarz, et al., J. Res. Sci. Teach. 46, 632 (2009). 